\title{
Preserved speech variant is allelic of classic Rett syndrome
}

\author{
Cristina De Bona ${ }^{1}$, Michele Zappella ${ }^{2}$, Giuseppe Hayek ${ }^{2}$, Ilaria Meloni ${ }^{1}$, Francesca Vitelli ${ }^{1}$, \\ Mirella Bruttini ${ }^{1}$, Roberto Cusano ${ }^{3}$, Paola Loffredo ${ }^{1}$, Ilaria Longo ${ }^{1}$ and Alessandra Renieri ${ }^{1}$
}

\author{
${ }^{1}$ Genetica M edica, Policlinico Le Scotte, Universitá di Siena; ${ }^{2}$ Neuropsichiatria Infantile, Policlinico Le Scotte, Siena; \\ ${ }^{3}$ Laboratorio di Genetica Molecolare, Istituto G Gaslini, Genova, Italy
}

Rett syndrome is a neurological disorder affecting predominantly females with regression loss of speech and purposeful hand use, after a few months of almost normal development. Postnatal microcephaly, hand dispraxia, stereotypic 'hand-washing' activities, ataxia, and abnormal breathing are among its most characteristic features. Another aspect of this disorder is growth failure. The preserved speech variant (PSV) shares with Rett syndrome the same course and the stereotypic hand-washing activities but it differs in that patients typically recover some degree of speech and hand use and usually do not show growth failure. Progressive scoliosis, epilepsy and other minor handicaps, usually present in Rett syndrome, are rare in the preserved speech variant. Here we explore the spectrum of mutations affecting the MECP2 gene in a group of 25 classic Rett syndrome girls and in three patients with the preserved speech variant. Among the Rett syndrome group, two novel mutational hot spots (R270X and R294X), four novel mutations, two novel small deletions, as well as the previously reported 806delG, R168X and R255X mutations, were identified in $20 / 25$ patients. Of note, among the preserved speech variants, two patients carry deletions of $41 \mathrm{bp}$ and $\mathbf{4 4} \mathrm{bp}$ each, which are strikingly similar to those observed in classic Rett syndrome. Our results confirm the presence of mutational hot spots in MECP2, broaden the spectrum of mutations, pinpoint additional mutational hot spots and establish that the preserved speech variant is indeed allelic of the classic form. Phenotype variability is only partially dependent on the kind of MECP2 mutation and other mechanisms such as skewed X-inactivation, and/or modifier gene effects should be investigated to explain the variable recovery in speech and hand use. European Journal of Human Genetics (2000) 8, 325-330.

Keywords: preserved speech variant; Rett syndrome; MECP2

\section{Introduction}

Rett syndrome (RTT, MIM No.312750) is a progressive neurological disorder affecting predominantly females. It has an early childhood onset and develops in four stages: 1 early onset stagnation, 2 rapid developmental regression, 3 pseudostationary period and 4 late motor deterioration. ${ }^{1}$ Some neurological features such as stereotypic hand-washing activ-

Correspondence: Alessandra Renieri MD, PhD, Medical Genetics, University of Siena, Policlinico Le Scotte, viale Bracci 2, 53100 Siena, Italy. Tel: + 390577 233303; Fax: + 390577 233325; E-mail: renieri@unisi.it

Received 22 December 1999; revised 21 January 2000; accepted 21 January 2000 ities, hand apraxia, and postnatal microcephaly are highly characteristic of this condition which is usually accompanied by a number of additional handicaps like epilepsy, abnormal breathing, and scoliosis.

Beside the classic form of RTT, a number of variants have been described since the mid-1980s: these include the infantile seizure onset type, ${ }^{2}$ the congenital form, ${ }^{3,4}$ the 'forme fruste' ${ }^{5}$ and late childhood regression. ${ }^{6}$ In the early 1990s, the preserved speech variant (PSV) was also described. ${ }^{7-9}$ In recent years the concept of a Rett complex was advanced, suggesting on clinical grounds that classic cases and variants probably belong to the same type of disorder. Among the variants, PSV is probably the most common; more than 30 cases have been described in the 
Department of Child Neuropsychiatry of Siena and a number of other cases have been observed in clinical practice. ${ }^{10,11}$

Very recently, molecular genetic studies established the exclusive genetic nature of this syndrome and pin pointed the MECP2 gene, encoding methyl-CpG binding protein 2, as the site of mutations. ${ }^{12}$ Despite the fact that the vast majority of cases are de novo mutations, the recurrence of both nonsense and missense mutations has been found, enabling the identification of mutational hot spots in the gene. ${ }^{13}$ In the rare familial cases, either somatic/germline mosaicism or skewed X-inactivation was found to be responsible for healthy adult carrier females. ${ }^{13} \mathrm{M}$ oreover, the recent paper by Wan et al showed that the MECP2 mutations are not limited to RTT and may be implicated in a broader phenotypic spectrum. ${ }^{13}$ These results prompted us to investigate both classic and variant forms of RTT. Findings observed in 25 classic RTT and three PSV girls are reported.

\section{Materials and methods \\ Patients}

Classic RTT Twenty-five sporadic cases of classic Rett syndrome have been analysed: the patients were aged between 2 years 8 months and 24 years 9 months (mean age 11 years 6 months). Eighteen patients were microcephalic, six normocephalic and one macrocephalic. Weight was below 2SD in 13 patients, normal in five, slightly above average in four, and above 2SD in three. Height was below 2SD in 15 patients, normal in nine and above 2SD in one. All the girls fulfilled the criteria given for this disorder by Trevathan et al. $^{14}$

Variants Three sporadic cases of the preserved speech variant (PSV) of the Rett complex have been analysed. They fulfilled the criteria given by Hagberg and Skjeldal for Rett variants. ${ }^{15}$

Case 32 (Figure1) has a healthy younger sister. Family history does not show the presence of a neurological disorder in three generations. After a normal pregnancy and delivery, her initial development was delayed. She was able to walk al one at 15 months, and speak her first words at the same age. At 18 months motor deterioration began with uncertain gait, hypotonus, and tremor in the hands accompanied by loss of their functional use. At the same time she lost the few words which she could speak, as well as her ability to relate. Some months later, stereotypic hand clapping and hand-washing activities appeared. In the following years, she recovered some degree of visual interaction with people and from 5 years of age she slowly began to recover some functional use of her hands, in which intention tremor and stereotypic activities remained present until 9 years of age when she was admitted to the Department of Child Neuropsychiatry of Siena. Her head appeared large with a circumference of $54 \mathrm{~cm}$, although within the limits of $2 \mathrm{SD}$. Her height $(152 \mathrm{~cm})$ and weight $(44 \mathrm{~kg})$ were above $2 \mathrm{SD}$. Kyphoscoliosis was present. She showed two café au lait spots over her right

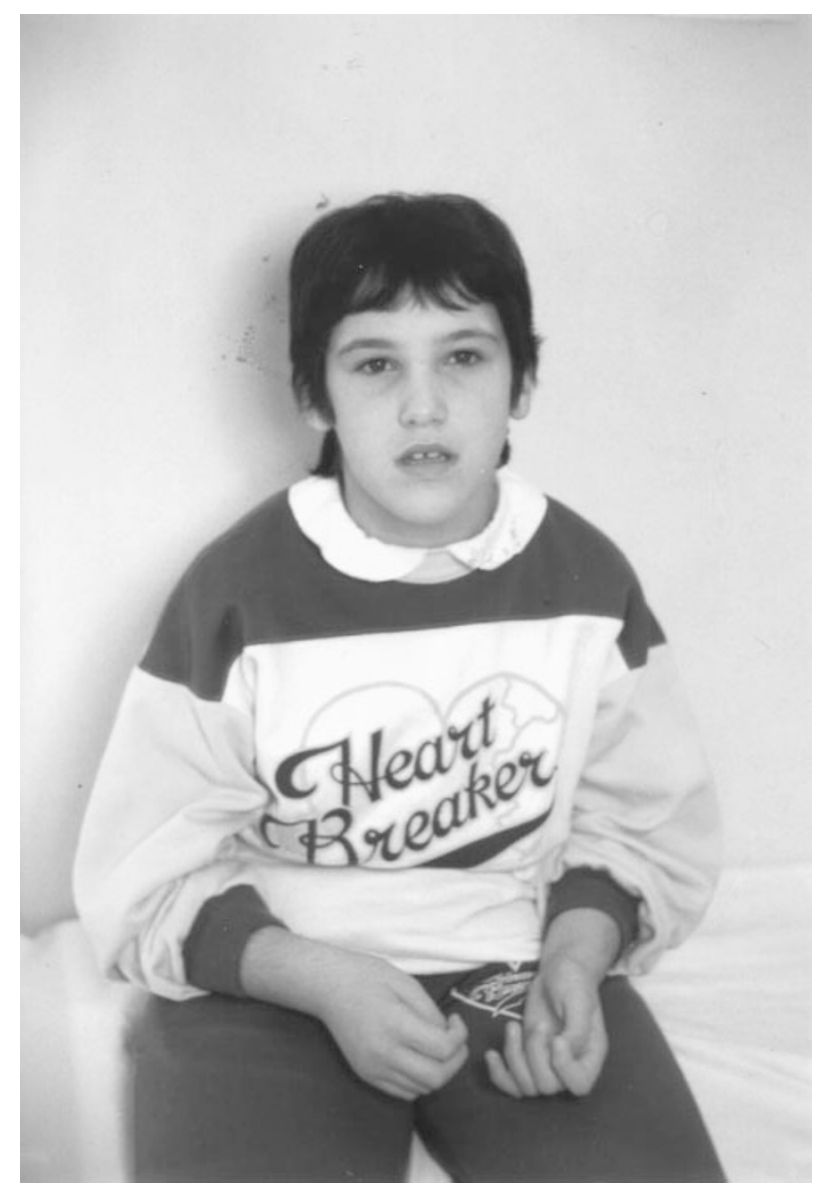

Figure 1 A PSV patient at 10 years of age (case 32). Note the large head, the coarse facial features and the plump appearance, in contrast to the microcephaly and the thin body structure typical of classic Rett girls.

thigh (each $4 \times 3 \mathrm{~cm}$ in size) and two other small depigmented spots on the abdomen. She had an ataxic gait and could say a few words in an echolalic manner. An EEG showed a well organised alpha activity with bursts of high voltage $2-3 \mathrm{c} / \mathrm{s}$ activities which were more evident in sleep. In the following years, her speech became more complex with the appearance of short phrases, pronounced in the second and third person, and echolalic. From age 10 there was a slow motor deterioration and now at 22 years she is in a wheel chair, maintains her stereotypic hand activities and can still say a few words, although less than at the beginning of adolescence. She has never had epileptic fits. Her head circumference is $55 \mathrm{~cm}$, her height $166 \mathrm{~cm}$, her weight $75 \mathrm{~kg}$. $A$ recent waking EEG shows the presence of generalised nonreactive $5 \mathrm{c} / \mathrm{s}$ activities.

Case 109 has already been fully described as case report (casel-12) in Zappella et al. ${ }^{11}$ At present the patient is 13 years old, is in the third stage and her conditions have remained essentially the same as in the description given. ${ }^{11}$ She is an obese girl with a high kyphosis, has continuous stereotypic 
hand-washing activities, speaks in short phrases in the second and third person with echolalias, and has a mental age close to 3-4 years. She also has flat feet, genu val gum and a dyspraxic gait. She has a large head circumference $(56.5 \mathrm{~cm})$, albeit within $2 \mathrm{SD}$. A recent waking EEG shows a basic rhythm of $8 \mathrm{c} / \mathrm{s}$ with bursts of $6-7 \mathrm{c} / \mathrm{s}$ activities: she has never had epileptic seizures.

The third case of PSV was already described in $1994 .^{8}$ The patient is now age 19 and her conditions have not changed since the original description. She is phenotypically similar to the above two reported cases: a girl with a large head, at the upper limits of 2SD deviations when initially seen at age 11, with a kyphoscoliosis of moderate degree, flat feet, genu valgum and a dyspraxic gait with persistent handwashing activities and a hand dyspraxia which, however, allows her to do simple drawings, and a mental age close to 4 years. She is able to speak in short phrases in the second and third person with frequent echolalias. She has never had epileptic fits.

\section{Molecular analysis}

DNA was extracted using standard procedures from the blood of probands and, where available, parents and siblings. PCR amplification of the coding portion of MECP2 was performed by using the published primers with some modifications. ${ }^{12}$ Primers for the exon 1 and for the most 3 ' portion of exon 3 were redesigned as follows: exon $1 \mathrm{~F}=5^{\prime}$ tactttgtctatgtgtttatc $3^{\prime}$ and $1 R=5^{\prime}$ ggcacagttatgtctttag 3'; exon 3: 3CF2 = 5' caggtcatggtgatcaaacg $3^{\prime}, \quad 3 C R 2=5^{\prime}$ actctgagtggtggtgatg 3', 3DF2 = 5 ggactgaagacctgtaagag 3', 3DR2 = 5'catggaggatgaaacaatgtc $3^{\prime}, \quad 3 E F 2=5^{\prime} \quad$ cttgagcagcagcgtctg $3{ }^{\prime}, \quad 3 E R 2=5^{\prime}$ cttcaacctgactgtgcttg $3^{\prime}$. For exon amplification, genomic DNA (100 ng) was denatured at $97^{\circ} \mathrm{C}$ for $5^{\prime}$, mixed with $10 \times$ buffer, $0.5 \mu \mathrm{m}$ primers, $200 \mu \mathrm{m}$ dNTP, $1 \mathrm{U}$ Taq polymerase in a final volume of $25 \mu \mathrm{l}$, and cycled $35 \times$ at $94^{\circ} \mathrm{C}$ for $1 \mathrm{~min}, 54^{\circ} \mathrm{C}$ (exon 1 ) or $58^{\circ} \mathrm{C}$ (exons $3 \mathrm{C}$ and $3 \mathrm{D}$ ) or $60^{\circ} \mathrm{C}$ for $1 \mathrm{~min}$ (exon $3 \mathrm{E}$ ), $72^{\circ} \mathrm{C}$ for $1 \mathrm{~min}$, and finally incubated at $72^{\circ}$ for $5 \mathrm{~min}$.
SSCP technique was performed as reported elsewhere ${ }^{16}$ with some modifications. Precast gradient polyacrylamide gels (12.5-2\%) were used in the GenePhor electrophoresis unit (Amersham-Pharmacia-Biotech, Uppsala, Sweden) under a controlled temperature of $10^{\circ} \mathrm{C}$ and/or $20^{\circ} \mathrm{C}$. Automated fluorescent DNA sequencing was performed using Perkin-Elmer ABI373A equipment according to PRISM Dye Terminator and Dye Primer Cycle Sequencing chemistries on double strand plasmid templates.

\section{Results}

We analysed 25 cases of classic Rett syndrome and three cases of PSV by SSCP of the coding portion of the MECP2 gene. Among classic RTT, 11 different mutations were found in 20 out of 25 cases (Table 1 ). Two novel mutations R294X and R270X were each observed in four unrelated cases. The previously reported mutations $\mathrm{R} 168 \mathrm{X}$ and $\mathrm{R} 255 \mathrm{X}^{12,13}$ were observed in three and two unrelated cases, respectively (Figure2). All the above mutations are $\mathrm{C}$ to $\mathrm{T}$ transitions confirming the high CpG mutability at this locus.

Two novel $C$ to $G$ transversions were found: one at position 423 leading to replacement of a tyrosine with a stop codon (Y141X) and the other at position 674 leading to the substitution of the conserved proline225 for an arginine. A novel $G$ to $T$ transversion was found at position 398 leading to the replacement of the conserved arginine133 for a leucine.

A C to T transition was found at position 984 (Table 1). This mutation is not likely to be pathogenic since it does not cause an amino acid change (leucine is retained), a splice-site effect is not expected on the base of the surrounding sequences, and it was found in an R168X-bearing sample. Moreover, its presence in the dead healthy father cannot be excluded.

Remaining mutations were small deletions. In one case, the previously described $806 \mathrm{del} G$ was found. ${ }^{13}$ In addition, a novel single nucleotide del etion, 431delA which occurred in a stretch of four identical nucleotides, was found in another patient. Four very similar small deletions were found in two

Table 1 MECP2 mutations and normal variant

\begin{tabular}{|c|c|c|c|c|}
\hline Mutation & Effect on protein & Recurrence & Presence in other papers & Phenotype \\
\hline G398T & R133L & - & - & classic \\
\hline C423G & Y141X & - & - & classic \\
\hline 431delA & 144 shift, stop at 145 & - & - & classic \\
\hline C502T & $\mathrm{R} 168 \mathrm{X}$ & $3 \times$ & $7 \times$ Wan et $a^{13}$ & classic \\
\hline C674G & P225R & - & - & classic \\
\hline C763T & $\mathrm{R} 255 \mathrm{X}$ & $2 \times$ & $2 \times$ Amir et al, $^{12}$ Wan et $\mathrm{al}^{13}$ & classic \\
\hline C808T & $\mathrm{R} 270 \mathrm{X}$ & $4 \times$ & - & classic \\
\hline 806delG & 269 shift, stop at 288 & - & $1 \times$ Wan et al & classic \\
\hline С880T & R294X & $4 \times$ & - & classic \\
\hline 1157del41 & 386del14 aa, stop at 404 & - & - & PSV \\
\hline 1159del41 & 387del14 aa, stop at 404 & - & - & classic \\
\hline 1159del44 & $387 d e l 15$ aa, stop at 404 & - & - & PSV \\
\hline 1165del69ins21 & 389del23/ins3 aa, stop at 393 & - & - & classic \\
\hline \multicolumn{5}{|l|}{ Normal variant: } \\
\hline C984T & L328L & - & - & classic \\
\hline
\end{tabular}




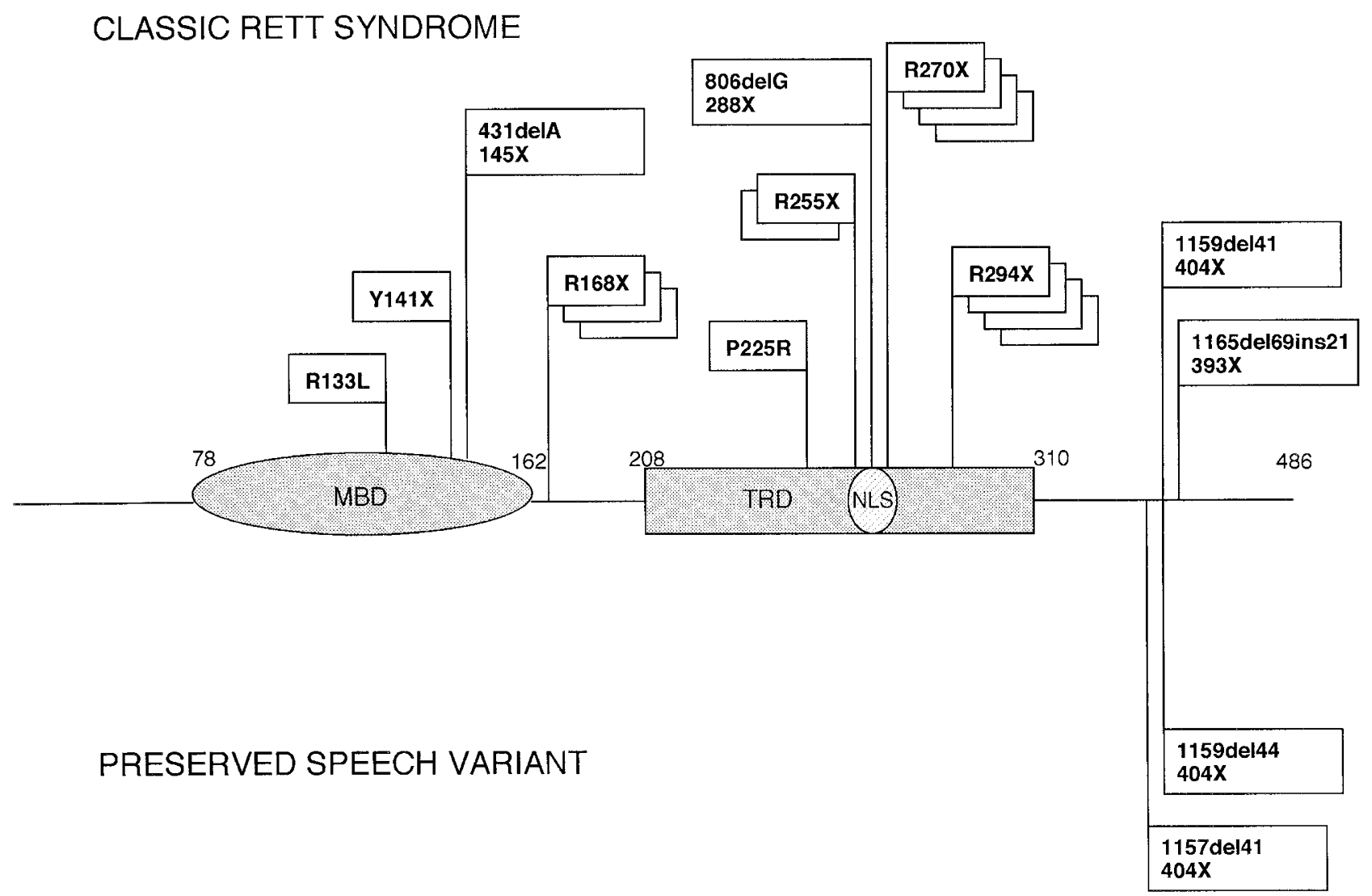

Figure 2 MeCP2 protein and mutations. The methyl-CpG-binding domain (MBD) and the transcriptional repression domain (TRD) which contains the nuclear localisation signals (NLS) are schematically depicted. Mutations found in classic RTT and in PSV are shown above and below the protein, respectively.

classic RTT patients and in two (out of three) preserved speech variants (case 32 and case 109, Figure 3). The deletion of $41 \mathrm{bp}$ (starting from nt1157) and the two deletions of $41 \mathrm{bp}$ and $44 \mathrm{bp}$ (starting from nt1159) led to the same frameshift and to protein truncation in position 404, after a short stretch of incorrect amino acids. These deletions fall near direct repeats 'ACCCC' and 'CCCCACC- - CCTGAGCCC' which may explain the occurrence of a hot spot for del etions (Figure3). The fourth deletion was more complex, resulting from a $69 \mathrm{bp}$ del etion and a 21 bp insertion (Figure 3c). These 21 bp do not belong to the MECP2 gene and a BLAST search in the non-redundant database revealed that they match perfectly with a single genomic sequence on chromosome $22 .{ }^{17}$ Analysis of sequences surrounding chromosome 22 suggests a mechan ism of non-homologous recombination for this complex deletion/insertion (Figure3).

Among the 25 classic RTT patients, no relevant phenotypic differences were found between the 20 girls with MECP2 mutations and the five girls without. In the same manner, no significant correlation between clinical course and mutation type was identified among the 20 mutation-bearing girls. The only notable findings were in the presence of macrocephaly ( $>2 \mathrm{SD}$ ) and slight overweight in the patient with the 1159del41 mutation, and slight overgrowth and overweight in the patient with the 1165del69ins21 mutation, who are otherwise typical RTT girls. De novo origin of all the above mutations was tested by analysis of both parents (except one case in which only the mother was available) and when present (16/20 cases) also of a healthy sibling. A negligible risk of recurrence was given in all cases, taking into account the possibility of a germline mosaicism. ${ }^{12}$

\section{Discussion}

Investigation of the coding region of the MECP2 gene in 25 classic Rett syndrome patients has allowed us to identify a mutation in about $80 \%$ of cases (20/25), which is significantly more than initially reported $\left(5 / 21^{12}\right)$ and also significantly higher than subsequently reported $(50 \%) .{ }^{13}$ All recurrent nucl eotide substitutions found in our cases are $C$ to T transitions in CpG dinucleotides, reinforcing the proposed mechanism of hypermutability at $\mathrm{CpG}$ sites. Moreover, our results confirm the prediction of Wan et al regarding arginine in position 270 as one of the potentially important mutation sites. ${ }^{13} \mathrm{~A}$ noteworthy result of our SSCP screening is the 
finding that small out-of-frame deletions contribute significantly to the mutational spectrum that inactivates the MECP2 gene. Peculiar features in the flanking DNA, such as direct/inverted repeats or symmetric sequences, are responsible for endogenous mutagenesis mechanisms. ${ }^{18}$ In our deletions, the model of 'polymerase slippage' on direct repeats appears to be the most likely mechanism (Figure3).

Except for R133L and P225R, most mutations identified in this study cause protein truncation. MeCP2 works in the nucleus where it selectively binds methylated CpG dinucleotides and mediates transcriptional repression through interaction with histone deacetylase and the corepressor SIN3A. Although we cannot exclude the possible translocation of an abnormal protein into the nucleus in those cases with missense mutations or mutations affecting the C-terminal portion, (since a putative second nuclear translocation signal is present near position 173), for the Y141X mutation, nuclear translocation is very unlikely. This confirms that the most likely consequence of mutations found in MECP2 gene may be a loss of function instead of a gain of function. ${ }^{12,13}$

The absence of causative mutations in $5 / 25$ cases may be attributed either to the limited sensitivity of SSCP analysis or to the presence of mutations in the long $3^{\prime}$ untranslated region of $\mathrm{MECP} 2$. In fact, the strong evolutionary conservation of this region suggests that it may play a role in the regulation and/or processing of the gene. Moreover, given

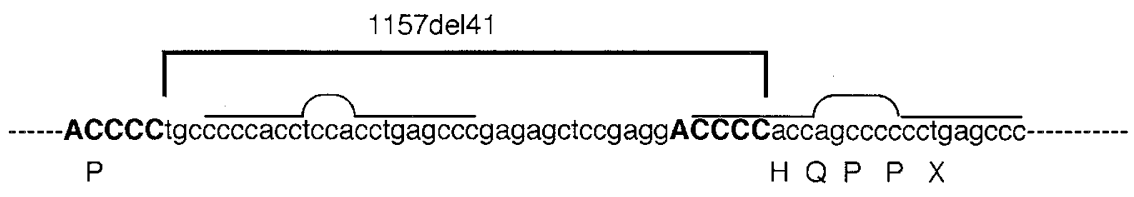

PSV case 109

a)

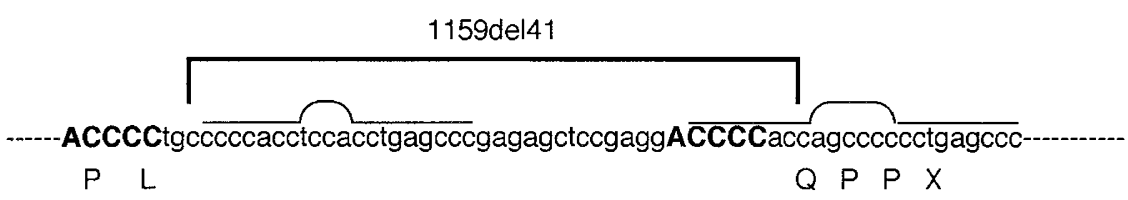

classic RS

b)

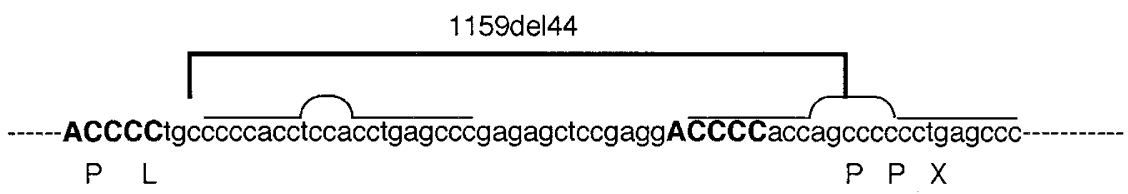

PSV case 32

c)

d)

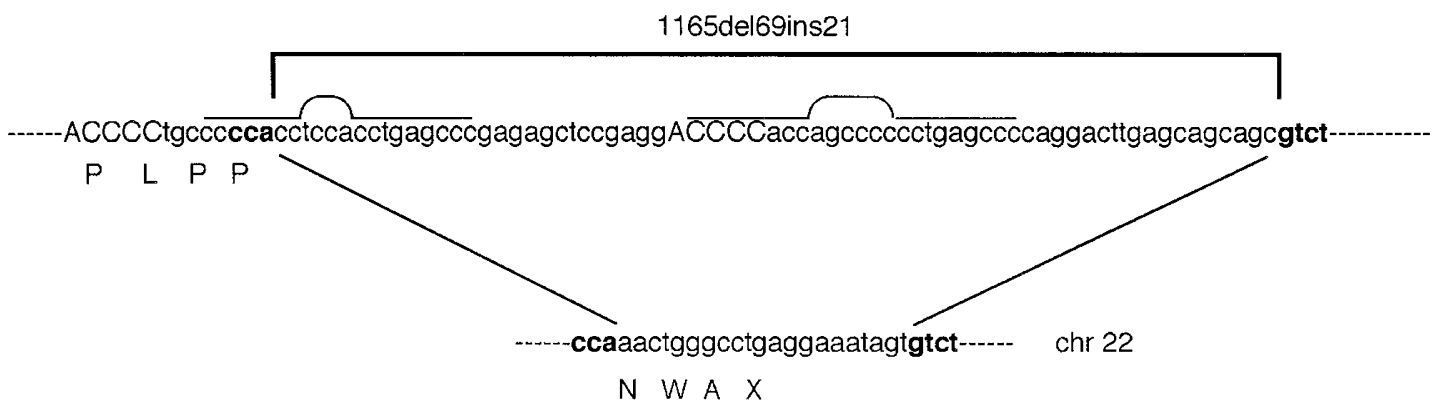

classic RS

Figure 3 Mechanism of small deletions and predicted mutated proteins. During 'polymerase slippage', the newly synthesised strand containing one copy of direct repeats 'ACCCC' (in bold) may separate from the template and reanneal to the subsequent copy, leaving out one repeat and the intervening sequence, to generating the 1157del41 mutation (a). Mutation 1159 del41 (b) and 1159del44 (c) may arise by imprecise cutting and/or joining mechanisms. Other direct repeats which may have a role in the deletions are overlined (ccccacc - - - cctgagccc). In panel d, the chromosome22 sequence, from genomic clone RP11-395L14, locus HSA395L14 (nts82446-82466), is surrounded by short stretches 'cca' and 'gtct' (in bold) which perfectly match the MECP2 sequences suggesting a role as putative homology arms. Deletion effects on the protein are represented by one-letter abbreviation for amino acids under the nucleotide sequences. Short stretches of abnormal amino acids, (H)Q)PPX and NWAX, are added before protein truncation. 
our finding of small deletions as a frequent mutational event in Rett syndrome, the presence of larger del etions cannot be excluded and del etions exceeding $300 \mathrm{bp}$ (the average length of amplified segments) may be lost using the current method of detection by amplification of the normal allele alone. Overall, the presence of an additional candidate gene seems less likely.

A major result of our analysis is the finding of MECP2 mutations in PSV. This is a disorder which has some el ements in common with classic RTT: it develops in four consecutive stages and it includes the presence of hand dyspraxia and similar stereotypic hand activities in the second and the beginning of the third stage. In contrast, in the following years there is a slow improvement in the use of hands and speech and usually by the second half of the first decade these girls are able to use a spoon and fork, to draw and to speak, initially with words, subsequently in short phrases based on the use of the second and third person and with frequent echolalias. Their behaviour fulfils the characteristics of an autistic disorder. Additional handicaps such as microcephaly, epilepsy, and scoliosis are present only in a minority of cases and the somatic phenotype is often marked by obesity and/or overgrowth (Figure 1). It is worth emphasising that, in contrast, growth failure is one of the hallmarks of the classic Rett syndrome. ${ }^{19}$ Our previous finding of a classic RTT and a PSV case within the same family ${ }^{7}$ strongly suggested that they may represent a variable spectrum of the same genetic defect. ${ }^{20}$ Our paper establishes that classic RTT and PSV are indeed allelic. This result is not surprising given the wide variability of phenotypes associated with MECP2 alterations. $^{13}$

Noteworthily, the mutations found in the PSV cases are frame-shift deletions in the $3^{\prime}$ end of the coding region, downstream of the transcription repression domain. The presence of two very similar mutations in two cases of classic RTT prevents the establishment of a clear correlation between the kind of mutations and the severity of the phenotype. However, since no PSV cases with mutations affecting the transcription repression domain or the methylCpG-binding domain have been observed, it cannot be excluded that mutations resulting in the milder PSV phenotype are confined to the region downstream of the two functionally important domains. The occurrence of both classic RTT and PSV causing mutations in the downstream deletion hot spot could be explained by skewed $X$-inactivation and/or modifier gene effects. In the light of this hypothesis, the slight overweight and large head in the RTT patient with the 1159 del 41 mutation and the slight overgrowth and overweight in the RTT patient with the 1165del69ins21 mutation might be considered suggestive of the physical PSV phenotype. Analysis of more PSV cases is needed to better address this issue.

Given the above results, we conclude that phenotype variability in Rett complex is only partially dependent on the kind of MECP2 mutation. It is conceivable that other mechanisms such as skewed $\mathrm{X}$-inactivation and/or modifier gene effects play a synergetic role to explain the remarkable recovery in speech and hand abilities in PSV.

\section{Acknowledgements}

This work was supported by a grant to AR from the Emma e Ernesto Rulfo foundation.

\section{References}

1 Hagberg BA: Rett Syndrome - Clinical and Biological aspects. McKeith Press: London, 1993.

2 Hanefeld F: The clinical pattern of the Rett syndrome. Brain Devel 1985; 7: 320-325.

3 Nomura Y, Segawa M, Hasegawa M: Rett syndrome - an early cathecolamine and indolamine deficient disorder? Brain Devel 1985; 7: 334-341.

4 Rolando S: Rett syndrome: report of eight cases. Brain Devel 1985; 7: 290-296.

5 Hagberg B, Rasmussen P: 'Forme fruste' of Rett syndrome-a case report. Am J Med Genet 1986; 24: 175-181.

6 Gillberg C: The borderland of autism and Rett syndrome: five case histories to highlight diagnostic difficulties. J Autism Devel Disord 1989; 19: 545-559.

7 Zappella M: The Rett girls with preserved speech. Brain Devel 1992; 14: 98-101.

8 Zappella M: Rett syndrome-like hand washing, developmental arrest and autistic symptoms in two Italian girls. Eur Child Adolesc Psychiatr 1994; 3: 52-56.

9 Skjeldal OH, von Tetzchner S, Jacobsen K, Smith L: Rett syndrome - distribution of phenotypes with special attention to the preserved speech variant. Neuropediatrics 1995; 26: 87.

10 Zappella M: The preserved speech variant of the Rett complex: a report of 8 cases. Eur Child Adolesc Psychiatr 1997; 6: 23-25.

11 Zappella M, Gillberg C, Ehlers S: The preserved speech variant: a subgroup of the Rett Complex: a clinical report of 30 cases. J Autism Devel Disord 1998; 28: 519-526.

12 Amir RE, Van den Veyver IB, Wan M, Tran CQ, Francke U, Zoghbi HY: Rett syndrome is caused by mutations in X-linked MECP2, encoding methyl-CpG-binding protein 2. Nat Genet 1999; 23: 185-188.

13 Wan M, Lee SS, Zhang $X$ et al: Rett syndrome and beyond: recurrent spontaneous and familial MECP2 mutations at CpG hotspots. Am J Hum Genet 1999; 65: 1520-1529.

14 Trevathan E, Moser HW: Diagnostic criteria for Rett syndrome. Ann Neurol 1988; 23: 425-428.

15 Hagberg BA, Sjkeldal OH: Rett variations: a suggested model for inclusion criteria. Pediatr Neurol 1994; 11: 5-11.

16 Renieri A, Bruttini M, Galli L et al: X-linked Alport syndrome: an SSCP-based mutation survey over all 51 exons of the COL4A5 gene. Am J Hum Genet 1996; 58: 1192-1204.

17 Altschul SF, Gish W, Miller W, Myers EW, Lipman DJ: Basic local alignment search tool. J Mol Biol 1990; 403-410.

18 Krawezak M, Cooper DN: Gene deletions causing human genetics disease: mechanisms of mutagenesis and the role of the local DNA sequence environment. Hum Genet 1991; 86: 425-441.

19 Schultz RJ, Glaze DG, Motil KJ et al: The pattern of growth failure in Rett syndrome. Am J Dis Child 1993; 147: 633-637.

20 Pini G, Milan M, Zappella M: Rett syndrome in northern Tuscany (Italy): family tree studies. Clin Genet 1996; 50: 486-490. 